\title{
Memória emocional: aplicação coletiva em universitários
}

\author{
Danilo Assis Pereira* \\ Tatiane Kanzler Barbosa Nunes**
}

\begin{abstract}
RESUMO - Este artigo buscou replicar a adaptação do teste de memória emocional de Frank e Tomaz (2000), verificando a viabilidade da aplicação coletiva em jovens. Participaram da pesquisa 63 universitários, com idade média de 23,3 anos. Os participantes foram divididos em dois grupos: $\mathrm{E}$ - emocional e $\mathrm{N}$ - neutro. Foram apresentados dois filmes, com 11 slides cada, sendo um com carga emocional e outro sem carga emocional. Na primeira etapa, os participantes pontuaram o quão emocional era considerado o filme apresentado. Na segunda etapa, realizada sete a dez dias depois, os participantes responderam um Teste de Reconhecimento de 65 itens para avaliar a recuperação da memória da estória apresentada. Os resultados foram similares ao de Cahill e McGaugh (1995) e de Frank e Tomaz (2000), mostrando uma diferença significativa para o slide 8 , entre ambos os grupos. Ainda, pôde-se fazer algumas sugestões para possíveis modificações ao Teste de Reconhecimento.
\end{abstract}

Palavras-chave: memória emocional, memória, processamento emocional, teste de reconhecimento.

\section{Emotional memory: collective application in undergraduate students}

\begin{abstract}
This paper replied an adaptation of Frank and Tomaz (2000) emotional memory test, verifying its viability of collective application in young. Sixty-tree undergraduate had participated, mean age of 23.3 years-old. Participants were divided in two groups: $\mathrm{E}$ - emotional, and $\mathrm{N}$ - neutral. Two eleven slides stories were showed, one emotional and one neutral. In the first phase, participants were told to rate the story emotional level. The second phase was accomplished seven days later, and participants were told to answer a 65 items recognition test
\end{abstract}

*Professor de Psicologia do UniCEUB. Psicólogo clínico no IBAC. Pesquisador-orientador do projeto PIC-UniCEUB. Contatos através do e-mail: danilo.assis@uniceub.br **Graduanda em Psicologia (7o. semestre) do UniCEUB. Voluntária do PIC/UniCEUB. Esta pesquisa é parte do Projeto de Iniciação Científica (PIC-UniCEUB) intitulado Memória Emocional em Idosos Residentes em Asilos do DF. Agradecemos ao prof. Carlos A. Tomaz (UnB) pelas sugestões e incentivos na realização do projeto. Agradecemos ainda às alunas de psicologia do UniCEUB, Adriana Gebrin e Vanessa Nery, pelas importantes contribuições. Esta pesquisa contou com apoio do LaboCIEN - UniCEUB. 
to evaluate their memory of the story. Results were similar to Cahill and McGaugh (1995), showing a significant difference to slide 8 in both groups. Some suggestions were made to possible changes in the recognition test.

Key words: emotional memory, memory, emotional processing, recognition test.

Alguns estudos sobre as relações entre emoção e memória, propõem que algumas características particulares da informação podem desencadear uma resposta emocional que varia segundo seu valor, positiva ou negativa, e segundo a propriedade "alertante" do estímulo, de neutro a excitatório. (Lang, 1995). A propriedade da ativação emocional poderia produzir, em alguns casos, incremento da retenção mnemônica e em outros casos uma diminuição de tal retenção. Alguns dos trabalhos sobre a retenção mnemônica foram realizados tanto em sujeitos normais (Cahill \& McGaugh, 1995, 1998; Taylor, Liberzon \& Fig, 1998) como em sujeitos com lesão cerebral (Adolphs, Cahill, Schul \& Babinsky, 1997; Frank \& Tomaz, 2003; Hamann, Cahill \& Squire, 1997 a; Hamann, Cahill, McGaugh \& Squire, 1997b; Kilpatrick \& Cahill, 2003). Os autores sugerem que ativação emocional produzida por algumas situações implementadas nos trabalhos mencionados pode promover um aumento da memória declarativa (McGaugh, 1990; McGaugh, McIntyre \& Power, 2002) inclusive em pacientes amnésicos (Hamann e cols., 1997). Por outro lado, também tem sido visto que experiências emocionais intensas consideradas como traumáticas podem causar amnésia psicogênica permanente (Baddeley, 1999).

A escolha das medidas de memória e instrumentos de investigação para esse tipo de estudo chega a ser um desafio em termos de validade interna e externa porque muitos dos estímulos utilizados para os sujeitos considerados controles, usualmente diferem dos utilizados com sujeitos considerados experimentais (Cahill \& McGaugh, 1995; Frank \& Tomaz, 2000). As diferentes características dos materiais podem confundir o efeito principal e limitar a generalização (Frank \& Tomaz, 2000).

Adicionalmente, os fatores culturais também podem afetar a valoração das experiências emocionais (Mauro, Sato \& Tucker, 1992). No entanto, é importante selecionar instrumentos que estejam validados para uma população em particular e corrigir, o melhor possível, as interferências derivadas das ferramentas utilizadas.

Diferentes instrumentos e estímulos têm sido usados em estudos de memória emocional, por exemplo, fotos "alertantes" (impactantes) e "neutras" (Cahill, 1999; Taylor e cols., 1998), expressões faciais emocionais (Adolphs e cols., 1999; Dolan, Fletcher, Morris, Kapur, Deakins \& Frith, 1996; Morris e col., 1998), slides com conteúdos emocionais opostos (Cahill e cols, 1996; Lane, Reiman, Ahern, Schwartz \& Davidson, 1998) e estórias visuais narradas (Cahill \& McGaugh, 1995; Lezak, 1995). Boa parte dos estudos obtidos com os protocolos dos trabalhos anteriores se reproduz qualitativamente quanto à potencialização mnemônica induzida pelos 
conteúdos emocionais alertantes.

Cahill \& McGaugh (1995) conceituam que as estórias utilizadas se assemelham de maneira rigorosa, o que facilita o controle do efeito principal para o examinador, isto é, o efeito do conteúdo emocional e não da novidade do material ou das diferentes características do estímulo audiovisual em condições experimentais ou o "efeito da estória". Resultados anteriores utilizando os protocolos de onde se derivam os implementados neste projeto têm sido obtidos em experimentos com adultos saudáveis (Cahill \& McGaugh, 1995); com pacientes que sofrem da enfermidade de Urbach-Wiethe, enfermidade degenerativa que afeta a amígdala (Adolphs e cols., 1997); em estudos com pacientes amnésicos (Hamann e cols., 1997), com pacientes epilépticos lobotomizados (Frank, Arruda \& Tomaz, 2001; Frank \& Tomaz, 2003) e a investigação dos efeitos noradrenérgicos e seu papel na memória (Cahill e cols., 1994). Em todos os casos aqui mencionados, pode-se evidenciar os efeitos da emoção alertante sobre o aumento da memória declarativa.

Na pesquisa realizada por Larry Cahill e James McGaugh (1998), onde se buscou investigar o papel da amígdala no armazenamento de conteúdos emocionais na Memória de Longo Prazo (MLP), dois filmes, compostos por dozes slides cada um, foram apresentados a oito sujeitos. Um dos filmes tinha um conteúdo emocional (E) e o outro um conteúdo neutro $(\mathrm{N})$. os sujeitos assistiram ao filme emocional e, após um período de três a sete dias, assistiram ao filme neutro. Durante a apresentação dos filmes, os sujeitos foram submetidos a sessões de tomografias realizadas por emissão de pósitrons (TEP). Após assistir a cada filme os sujeitos foram solicitados a classificar o grau de emoção do mesmo, usando para isso uma escala variando do 0 (zero) para conteúdo "nada emocional" e 10 (dez) para conteúdo "altamente emocional". As médias da emoção dos filmes encontrados foram 5,17 (EPM $\pm 0,73)$ para o filme emocional (E) e 3,09 (EPM \pm 0,69) para o filme não-emocional, considerado neutro $(\mathrm{N})$. Os resultados mostraram que houve uma recordação significativamente maior do filme emocional que do filme neutro, sendo 6,25 (EPM $\pm 1,06)$ o número de itens lembrados da estória emocional e 2,75 (EPM $\pm 0,67)$ o número de itens lembrados da estória neutra. A TEP mostrou uma correlação entre a ativação da amígdala direita - através da taxa metabólica de glicose - e a recordação dos itens da estória emocional $(r=0.93 ; P<0,1)$. Essa correlação não foi observada com a estória neutra.

No Brasil, Frank e Tomaz (2000) realizaram uma pesquisa onde demonstram um adaptação do teste de Cahill e cols. (1998). Participaram da pesquisa 46 sujeitos adultos, saudáveis, divididos em dois grupos. Foi apresentado aos grupos um filme composto por 11 slides, sendo o mesmo filme para ambos os grupos, diferindo apenas a narração gravada de uma estória acompanhando o filme. Um dos grupos assistiu ao filme com a narração de uma estória com um conteúdo emocional e o 
outro grupo assistiu ao filme com a narração de um conteúdo considerado emocionalmente neutro. $\mathrm{O}$ filme emocional conta a estória de um menino que vai com sua mãe visitar o pai em seu local de trabalho e sofre um acidente. $\mathrm{O}$ menino é levado ao hospital e é feita uma cirurgia de emergência. A estória pode ser dividida em três fases, sendo a segunda fase considerada a mais emocional (sobre o menino no hospital e a cirurgia das pernas). O filme neutro conta a estória do menino que vai com sua mãe visitar o pai em seu local de trabalho (hospital) e no caminho, vê um acidente de carro. No hospital, o menino é convidado para assistir uma simulação de emergência. Após assistirem ao filme, os sujeitos deveriam classificar o grau de emoção da estória, usando uma escala variando de 0 (zero) para conteúdo "nada emocional" e 10 (dez) para conteúdo "altamente emocional". A média foi de 7,34 $(\mathrm{EPM} \pm 0,29)$ para o grupo que assistiu à estória emocional e 5,17 $(\mathrm{EPM} \pm 0,51)$ para o grupo que assistiu à estória neutra. Os resultados mostraram que os indivíduos que assistiram ao filme emocional lembraram mais da estória - em particular da segunda fase - do que os indivíduos que assistiram ao filme neutro, sendo a pontuação do teste de recordação do grupo emocional 10,00 $(\mathrm{EPM} \pm 0,95)$ e a pontuação do teste de recordação do grupo neutro 6,78 (EPM $\pm 0,79)$, com $t=$ 2,59 e $P<0,01$.

Mais recentemente, Oliveira, Garrido, Cotes, Sarmiento e Tomaz (2003, no prelo) adaptaram o Teste de Memória Emocional na Colômbia com uma amostra de 62 sujeitos. Os sujeitos que observaram a estória emocional recordaram mais detalhes da estória $(t=3,672 ; P=0,006)$ e obtiveram uma melhor pontuação no questionário de reconhecimento que o grupo que observou a estória com conteúdo neutro ( $t=$ $2,8000 ; P=0,007)$.

\section{Justificativa}

A presente pesquisa faz parte de um Projeto de Iniciação Científica fomentada pelo Centro Universitário de Brasília (PIC - UniCEUB). O objetivo desta pesquisa é verificar a viabilidade da aplicação do Teste de Memória Emocional (Frank \& Tomaz, 2000) de forma coletiva.

\section{Método}

\section{Sujeitos}

A primeira parte da pesquisa contou com uma amostra de 69 participantes voluntários saudáveis (grupo controle), provenientes do curso de Psicologia de uma faculdade do Distrito Federal, que contribuíram para a coleta da primeira etapa 
do experimento, sendo 09 do sexo masculino e 60 do sexo feminino, com idades entre 19 e 53, média de 24,3 anos. Porém, este número foi reduzido para 63 participantes na segunda etapa, sendo 08 do sexo masculino e 55 do sexo feminino, com idades entre 18 e 53, média de 23,2 anos. Para participar da segunda etapa, os voluntários deveriam ter participado também da primeira. Os participantes foram classificados em dois grupos: o grupo E (emocional) e o grupo $\mathrm{N}$ (neutro). Os participantes foram escolhidos de forma pseudo-aleatória para compor um grupo ou outro. A condição era que ficasse metade do número inicial de voluntários para participar do experimento numa condição e a outra metade na segunda condição. Os próprios participantes decidiam se fariam parte do primeiro grupo ou do segundo, porém sem saber do que se tratava. A coleta estendeu-se por dois dias em turmas diferentes de dois turnos, na primeira etapa do experimento, tendo um intervalo de sete dias para a coleta da segunda etapa.

\section{Estímulos}

Dois filmes, com seqüência de 11 slides cada, continham estórias de um garoto que saiu de casa com a mãe para visitar o pai num laboratório de um hospital (ver Frank \& Tomaz, 2000 para maiores detalhes). A seqüência de slides era acompanhada pela fala de um narrador através de um alto-falante. Um dos filmes foi considerado como possuindo carga emocional (E) e o outro sem informações emocionais, considerado neutro $(\mathrm{N})$. A duração de cada filme era de aproximadamente dois minutos. A apresentação de cada slide era de aproximadamente 15 segundos, passando automaticamente para o slide seguinte após este período, totalizando 2 min e $45 \mathrm{~s}$, aproximadamente (ver CD-ROM Memória Emocional, Tomaz, 2002).

A apresentação dos filmes foi feita através de computador portátil multimídia e data show, projetado em uma tela branca de dimensões 1,4 $\mathrm{m}$ de altura e 1,7 $\mathrm{m}$ de largura em um auditório escuro. Os participantes ficavam sentados de frente para o estímulo visual. A distância entre a tela e os sujeitos era de aproximadamente 2,5 $\mathrm{m}$ a $6 \mathrm{~m}$. O som apresentado era realizado por amplificadores acústicos estéreos situados próximos ao estímulo visual. A sonoridade foi considerada adequada para o ambiente.

\section{Procedimento}

A obtenção dos dados foi feita coletivamente, com aproximadamente 10-15 participantes por vez. Foi instruído aos participantes que seria apresentada uma seqüência de slides, simultaneamente à fala de um locutor, numa duração total de dois minutos. Foi dito que seria realizada uma coleta após a apresentação para 
avaliar a atenção dos participantes perante o filme apresentado. Cada grupo foi exposto apenas a um filme, sendo $\mathrm{E}$ ou $\mathrm{N}$. A escolha do filme foi feita de forma pseudo-aleatória.

A pesquisa foi realizada em duas etapas. Na primeira etapa, logo após a apresentação do filme, os participantes pontuaram numa escala emocional (variando de 0 a 10, onde 0 corresponderia a "nada ou nenhum pouco emocional" e 10 a "totalmente ou completamente emocional") como consideravam aquele filme. Foi pedido aos participantes que nada comentassem sobre nenhuma parte do filme ou sobre suas pontuações com outras pessoas, para não interferir nos dados. $\mathrm{Na}$ segunda etapa, realizada sete dias depois, os alunos responderam um questionário (Teste de Reconhecimento) com 65 itens que avaliasse a memória dos participantes em relação ao filme visto. O Teste de Reconhecimento era diferente para os dois grupos (ver Anexo). Em nenhum momento foi dito que se tratava de uma pesquisa sobre memória.

\section{Resultados}

A pontuação média obtida, na primeira etapa da pesquisa, foi de 6,41 (EPM +/- 0,40) para o filme emocional (E) e de 1,97 (EPM +/- 0,31) para o filme neutro (N), conforme pode ser visto na Figura 1 (ver Anexos). Foi calculado o teste $t$ de amostras independentes, utilizando o pacote SPSS 11.5, obtendo significância $(t=8,411 ; P$

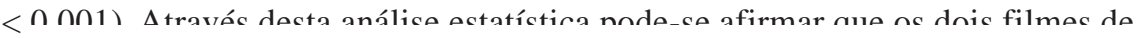
fa

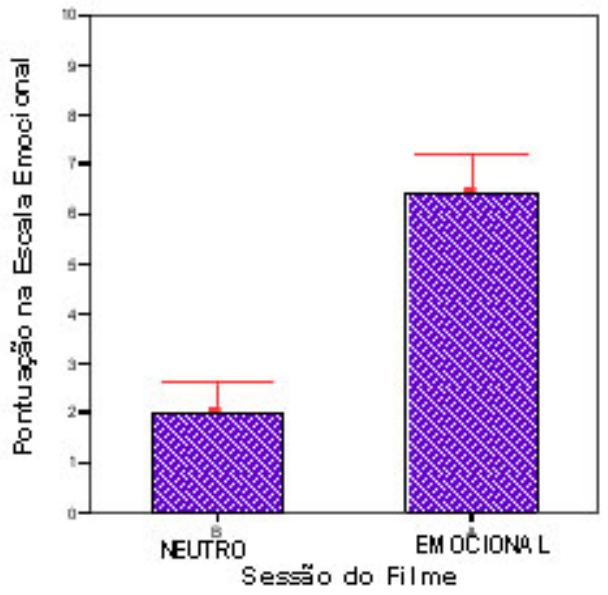

Figura 1 - Pontuação na escala emocional para os filmes neutro e emocional. 
Na segunda etapa da pesquisa, os sujeitos responderam um teste de reconhecimento de 65 itens que avaliasse a memória de cada slide apresentado na semana anterior. Os dados foram analisados através do teste $t$ para amostras independentes, verificando se haveria diferenças significativas entre os slides recordados no grupo emocional e no grupo neutro. Não foram encontrados diferenças significativas, com exceção do item 8 (ver Figura 2), slide onde mostra os pés do garoto já costurados (versão E) ou maquiados imitando ferimentos (versão N).

Te ito de Momorla

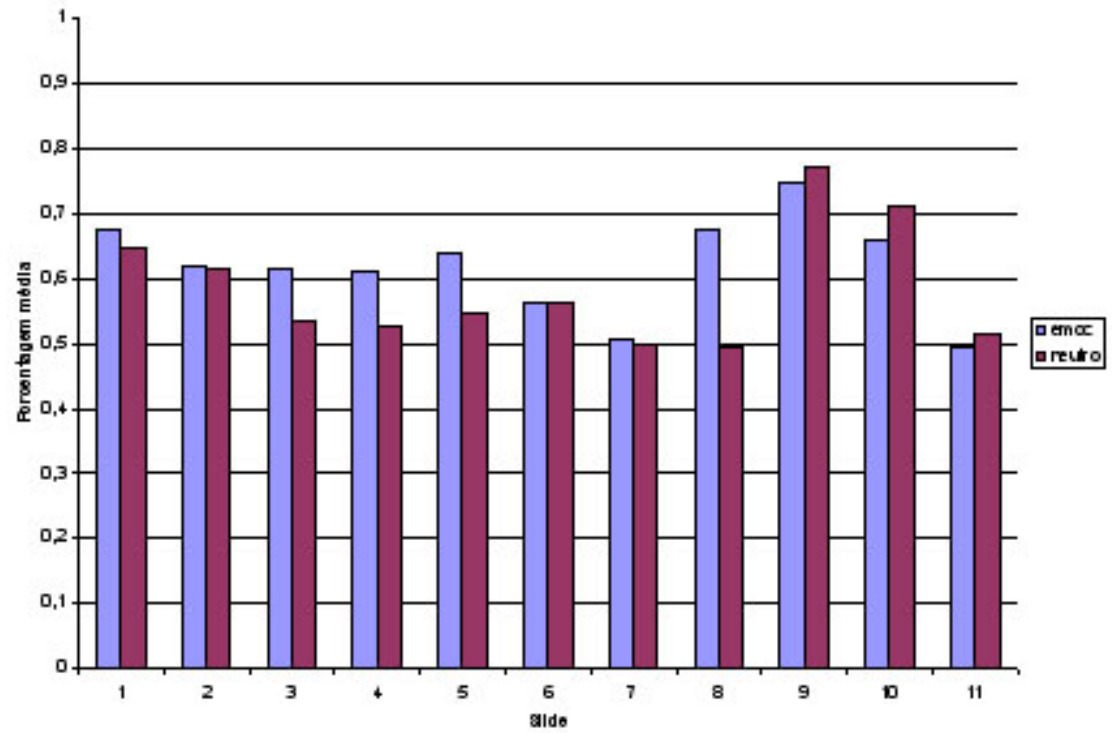

Figura 2 - Porcentagem média para os onze slides apresentados na estória emocional e os onze apresentados na estória neutra.

Neste slide, a média da porcentagem de acerto foi de 0,67 para a versão E; e 0,49 para a versão $\mathrm{N}(t=2,43$ e $P<0,02)$. Numa segunda análise, os slides foram separados em três fases: Fase 1, que correspondem aos slides 1 ao 4; Fase 2, que correspondem aos slides de 5 a 8; e Fase 3, que correspondem aos slides 9 a 11. As Fases 1 e 3 não diferem entre as versões, ficando esta diferença concentrada na Fase 2. Nenhuma das três Fases teve diferença significativa na porcentagem de acertos na versão emocional e na versão neutra, conforme observado na Figura 3. 


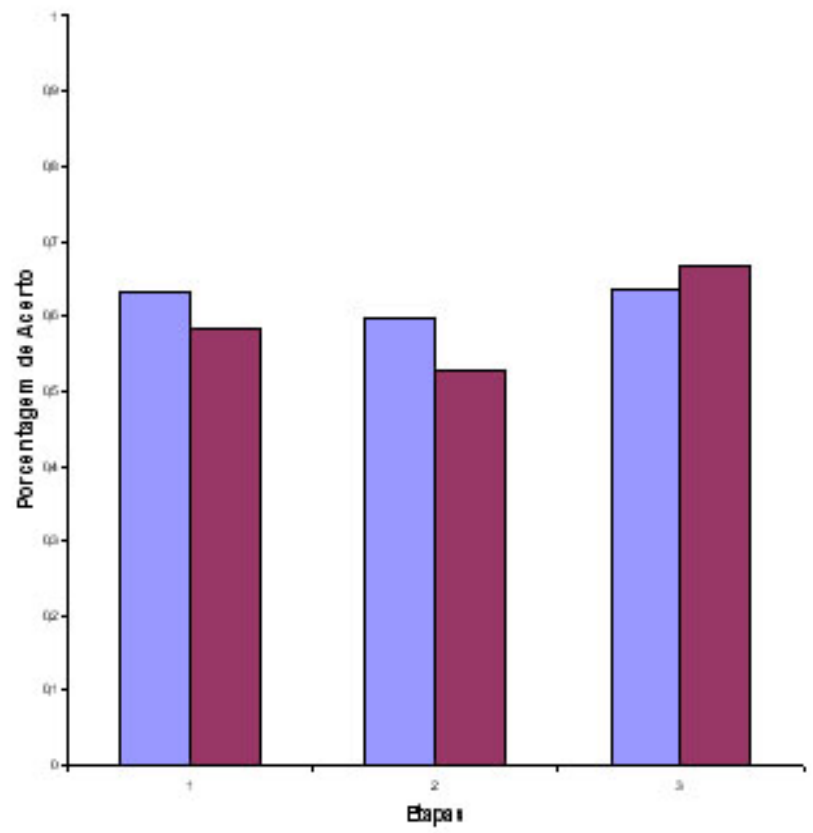

Figura 3 - Porcentagem de acerto das três etapas das estórias emocional e neutra.

\section{Discussão}

Os dados obtidos nas duas etapas deste experimento foram consistentes com os obtidos por Frank e Tomaz (2000). A diferença na forma de aplicação do teste, onde nesta pesquisa foi utilizada uma versão eletrônica, apresentada em datashow, e de coleta coletiva, mostrou ser completamente viável. A validação do experimento (escala emocional e teste de reconhecimento) em sua versão brasileira poderá ser realizada através de aplicação coletiva bem controlada. Para validação, será necessária a aplicação na comunidade, ampliando a variabilidade da idade, sexo, pessoas destras e canhotas, classe social e níveis de educação. Ainda, para a validação clínica em portadores de lesões ou deficiências no lobo temporal (mais especificamente a amígdala), os testes deveriam ser aplicados também neste grupo. Pode ser perfeitamente possível dar indícios de danos ou prejuízos em áreas da amígdala (mais especificamente da amígdala direita, conforme observado por Cahill e cols., 1996) observando o desempenho atingido ao responder o teste.

Os resultados da segunda fase desta pesquisa foram muito semelhantes aos encontrados por Frank e Tomaz (2000), onde o slide 8 teve uma diferença 
significativa. Porém, não conseguimos mostrar diferença para a Fase 2 da pesquisa, que incluiria os slides de 5 a 8, obtida no Teste de Recuperação Livre de Frank e Tomaz (2000).

As críticas levantadas por estes autores sugerem que o instrumento de avaliação (Teste de Reconhecimento) apresentava problemas semânticos e estruturais, o que parece ter sido observado também em nossa análise. O Teste de Reconhecimento necessita de ajustes para que possa descrever melhor os slides apresentados. Uma comissão de cinco juizes analisou o Teste de Reconhecimento em ambas as versões, E e N, e foram unânimes ao apontarem problemas nos itens seguintes:

\section{Versões Neutra e Emocional - Teste de Reconhecimento:}

Questão 1:4 - O que a mãe está fazendo?
a) Trancando o portão.
b) Amarrando o laço do sapato do filho.
c) Entrando no carro.
d) De pé em frente ao portão.

Comentário: Ficou ambígua a questão, colocando dúvidas entre a letra a) e d).

Questão 6:2 - Qual é a cor do hospital?
a) Verde.
b) Amarelo claro.
c) Marrom.
d) De duas cores.

Comentário: Os juizes concordaram que as cores estão imperceptíveis para este julgamento.

Questão 6:4 - Que parte do hospital se vê?
a) A entrada do hospital?
b) $\mathrm{O}$ muro e o portão.
c) Muitos andares e o telhado.
d) Todo o hospital.

Comentário: As letras a), c) e d) podem estarem perfeitamente corretas, pois tais imagens aparecem!

Questão 7:6-Qual é a expressão no rosto dele? 

a) Triste.
b) Alegre.
c) Normal.
d) Chocado.

Comentário: Dois juizes julgaram pela letra d) e três pela letra c). A diferença ocorreu devido ao tipo de estória, sendo maior a escolha pela letra c) no filme $\mathrm{N}$ e pela letra d) no filme E.

Questão 8:6 - Qual é a posição da pessoa/menino?
a) Deitado de bruços.
b) Deitado de costas.
c) Deitado de lado.
d) Sentado.

Comentário: As respostas b) e d) são possíveis.

Questão 9:3 - O que ela está levando na mão?
a) Sua bolsa.
b) Suas chaves.
c) Uma bola de futebol.
d) Nada.

Comentário: De fato ela não está levando nada na mão! Porém, no slide seguinte (10) aparece uma bola. A bolsa não está na mão, e sim no ombro da senhora.

Questão 9:4 - Ela está andando próximo a:
a) Uma delegacia de polícia.
b) Uma parada de ônibus.
c) Um ponto de taxi.
d) Um prédio.

Comentário: Nada foi dito a respeito. Há apenas um taxi ao fundo, que pode ser identificado pela placa.

Questão 11:6 - Qual o número do ônibus da parada onde ela está esperando?

Comentário: Na verdade o número é relativo à parada, e não à ônibus. 


\section{Conclusões}

A pesquisa realizada demonstrou ser viável a utilização coletiva do Teste de Memória Emocional traduzido e adaptado por Frank \& Tomaz (2000). Apesar de serem sugeridas algumas alterações no Teste de Reconhecimento, considerase que o mesmo tem uma estrutura bastante consistente. Sugere-se, para futuras pesquisas, verificar a memória emocional em idosos saudáveis e compará-los com idosos residentes em asilos do Distrito Federal, o que poderia possibilitar um maior conhecimento do desenvolvimento da memória emocional humana.

\section{Referências}

ADOLPHS, R., CAHILL, L., SCHUL, R. E BABINSKY, R. Impaired declarative memory for emotional material following bilateral amygdala damage im humans. Learning and Memory, 4, 291-300, 1997.

ADOLPHS, R., TRANEL, D., HAMANN, S., YOUNG, A. W., CALDER, A.J. PHELPS, E. A., ANDERSON, A., LEE, G.P. \& DAMASIO, A .R. Recognition of facial emotion in nine individuals with bilateral amygdala damage. Neuropsychologia, 37, 11111117, 1999.

BERTOLUCCI, P.H.F., BRUCKI, S.M.D., CAMPACCI, S.R. \& JULIANO, Y. O MiniExame do Estado Mental em uma população geral. Arquivos de Neuropsiquiatria, 52 (1), 1-7, 1994.

CAHILL, L. A neurobiological perpective on emotional influence on long-term memory. Seminars in Clinical Neuropsychiatry, 4, 266-273, 1999.

CAHILL, L. \& McGAUGH, J. L. Amygdaloid complex lesions differentially affect retention of tasks using appetitive and aversive reinforcement. Behavioral Neuroscience, 104, 532-543, 1990.

CAHILL, L. \& McGAUGH, J. L. A novel demonstration of enhanced memory associated with emotional arousal. Conciousness and Cognition, 4, 410-421, 1995.

CAHILL, L. \& McGAUGH, J. L. Mechanisms of emotional arousaland lasting declarative memory. Trends in Neuroscience, 21, 294-299, 1998.

CAHILL, L., HAIER, R.J., FALLONS, J., ALKIRE, M., TANG, C., KEATOR, D.,WU, J. \& McGAUGH, J.L. Amygdala activy at enconding correlated with long-term, free recall of emotional information. Proceedings of the National Academy of Science of the United States of America, 93, 8016-8021, 1996.

CAHILL, L., PRINS, B., WEBER, M \& McGAUGH, J.L. b- Adrenergic activation and memory for emotional events. Nature, 307, 702-704, 1994.

DOLAN, R.J., FLETCHER, P., MORRIS, J., KAPUR, N., DEAKIN, J.F.W. \& FRITH, C.D. Neural activation during covert processing of positive emotional facial 
expressions. Neuroimage, 4, 194-200, 1996.

FRANK J.E., ARRUDA, F. \& TOMAZ, C.A. Evaluation of emotional memory in patients with unilateral temporal lobectomy. Salud UIS , 3, 215-222, 2001.

FRANK J.E., \& TOMAZ, C.A. Enhancement of declarative memory associated with emotional content in a Brazilian sample. Brazilian Journal of Medical e Biological Research, 33, 1483-1489, 2000.

FRANK J.E., \& TOMAZ, C.A. Lateralized impairment of the emotional enhancement of verbal memory in patients with amygdala-hippocampus lesion. Brain and Cognition, 1-8. Article in press, 2003.

HAMANN, S.B., CAHILL L. \& SQUIRE, L.R. Emotional perception and memory in amnesia. Neuropsychology, 11, 104-113, 1997 a.

HAMANN, S.B., CAHILL L., McGAUGH, J.L. \& SQUIRE, L.R. Intact enhancement of declarative memory for emotional material in amnesia. Learning and Memory, 4, 301-309, 1997b.

KILPATRICK, L \& CAHILL, L.Modulation of memory consolidation for olfactory learning by reversible inactivation of the basolateral amygdala. Behavior Neuroscience, 1 , 184-188, 2003.

LANE, R. D., REIMAN, E. M., AHERN, G.L., SCWARTZ, G.E. \& DAVIDSON, R.J. Neuroanatomical correlates of happines, sadness and disgust. American Journal of Psychiatry, 154, 926-933, 1998.

LANG, P. The emotional probe. Studies of motivation and attencion. America Psycologist, 50, 372-385, 1995.

LEZAK, M. Neuropsychological assessment. ( ${ }^{\mathrm{a}}$ Ed.) New York: Oxford University Press, 1995.

MAURO, R., SATO, K., \& TUCKER, J. The role of appraisal in human emotions: A crosscultural study. Jounal of Personality and Social Psychology,62, 301-317, 1992.

McGAUGH, J.L. Significance and remenberance.: The role of neuromodulatory systems. Psychological Science, 1, 15-25, 1990.

McGAUGH, J.L., McINTYRE, C.K. \& POWER, E. Amygdala modulation of memory consolidation: Interaction with other brain systems. Neurobiology of Learning na Memory, 78, 539-552, 2002.

MORRIS, J.S., FRISTON, K.J., BUCHEL, C.D., FRITH, C.D., YOUNG, A . J., CALDER, A. J. \& DOLAN R.J. A neuromodulatory roel for the human amygdala in processing emotional facial expressions. Brain, 121, 47-57, 1998.

TAYLOR, S.F., LIBERZON, I., \& FIG, M. The effect of emotional content on visual recognition memory: APET acftivation study. Neuroimage, 8, 188-197, 1998. 